\title{
PERCEPCIÓN FEMENINA: VENTAJAS Y DESVENTAJAS DE LA COMPRA VIRTUAL
}

\section{FEMALE PERCEPTION: ADVANTAGES AND DISADVANTAGES OF VIRTUAL SHOPPING}

\author{
Yeily Marcela Ríos Quintana ${ }^{1^{*}}$ \\ Edward Fabian Moreno Mendoza ${ }^{2}$ \\ Cindy Paola Pineda López ${ }^{3}$
}

${ }^{1}$ Corporación de Gestión Empresarial, Investigativa y de proyectos ORCID: https://orcid.org/0000-0001-9884-9894

\author{
${ }^{2}$ Corporación de Gestión Empresarial, Investigativa y de proyectos-Colombia \\ ORCID: https://orcid.org/0000-0002-8558-6551, E-mail: morenomendozaef@ gmail.com \\ ${ }^{3}$ Corporación de Gestión Empresarial, Investigativa y de proyectos \\ ORCID: https://orcid.org/0000-0002-6149-7925, E-mail: cipapilo.04@ gmail.com
}

\section{Fecha de recibido: 03 de Julio de 2017}

Fecha aprobación: 15 de Julio de 2017

Resumen: Al momento de comprar por internet existen algunos tipos de factores que inciden en el proceso, ya que el cliente establece una evaluación indirecta con sus criterios específicos para saber si la empresa y el producto cumple y satisface totalmente sus expectativas y de esta manera genera confianza para ejecutar su adquisición. Las compras online no solo benefician a los compradores, sino también a los vendedores, más en épocas donde la dinámica virtual es tendencia. Se utilizó una investigación de tipo descriptiva con enfoque cuantitativo aplicado a las estudiantes del programa de Administración de Empresas, con los resultados se logró identificar ventajas y desventajas que al realizar las compras por internet. Conclusión: Las mujeres están interesadas en el desarrollo de la compra virtual a pesar de no interactuar con el producto, por otra parte, la conectividad y la desconfianza (Perdida del dinero) son las desventajas principales en el e-commerce.

Palabras claves: Compra virtual, Percepción, Factores de compra.

Abstract: At the moment of buying on the Internet there are some types of factors that influence the process since the client establishes an indirect evaluation with his specific criteria to know if the company and the product totally fulfills and satisfies his expectations and in this way generates confidence to execute his acquisition. Online shopping not only benefits the buyers, but also the sellers, more so in times where virtual dynamics is a trend. The results of this descriptive research with a quantitative approach applied to the students of the Business Administration program were used to identify advantages and disadvantages of making purchases over the Internet. Conclusion: Women are interested in the development of virtual shopping in spite of not interacting with the product. On the other hand, connectivity and distrust (loss of money) are the main disadvantages in e-commerce.

Keywords: Virtual Shopping, Perception, Buying Factors.

Autor para correspondencia.

Correo electrónico: morenomendozaef@ gmail.com

La revisión por pares es responsabilidad de la Universidad Francisco de Paula Santander Ocaña

Artículo bajo licencia CC BY-NC (https://creativecommons.org/licenses/by-nc/4.0/) 


\section{Introducción}

En la actualidad la internet se ha convertido en un plus y un factor de éxito en el comercio, ya que por medio de las compras virtuales el cliente y el ofertante tienen ganancias, puesto que es posible mantenerse en contacto desde la distancia y lograr ampliar la posibilidad de adquirir los productos. Antes de ejecutar la compra la persona indirectamente observa, critica y otorga opiniones de dicho bien o servicio que quiere alcanzar. El mundo del internet crece cada día más y abarca cada uno de los temas potencias tratados diariamente, como lo es la comunicación, el conocimiento científico, entre otras. Y por supuesto, el área económica no se podía quedar atrás, el movimiento del mercado también es visiblemente significativo y trascendental en el ámbito digital, ya que el comercio trabaja en esta actividad, aclarando que es uno de los grandes mandos en internet, por la razón de que existen factores benéficos que garantizan las ventajas de participar en este medio. La tecnología cada vez va fortaleciendo e incrementando su potencial. Kutchera, García, y Fernandez (2014) afirma que:

Internet no es solo un medio publiciario sino una plataforma de comercialización que abre nuevas oportunidades a las empresas y está cuestionando el modelo de negocio actual de bastantes de ellas (entretenimiento, equipo de cómputo y fotografía, servicios de viaje $\mathrm{y}$ otros muchos más).

A raíz de que el internet evoluciona constantemente, el vendedor también debe hacerlo, generando estrategias y adaptándose al conocimiento de algunos factores que inciden en la compra del cliente por medio online.
Cada vez son más los usuarios que quieren hacer parte de la compra por internet, pero como se dijo anteriormente, ellos son los encargados de decidir a qué empresa comprar y qué producto adquirir y para esto se basa en algunos principios primordiales para realizar tal selección. El cliente evalúa la página web y lo mide indirectamente a través de diez factores líderes en el mundo digital con respecto al cliente. El primero es la navegación sencilla, que exista facilidad al manejar la plataforma e intuición. La segunda es la buena ficha del producto $y$ disponibilidad del mismo, que se define como el deber de dar toda la información que se necesite saber del bien o servicio; Tercera la búsqueda personalizada, es importante facilitar filtros en la página web, ya que estos ayudan a que el producto que está buscando $\mathrm{X}$ o Y cliente sea encontrado más rápido. $\mathrm{El}$ cuarto se refiere a la determinación gastos de envío y devoluciones, el vendedor debe dejar en claro desde el principio las políticas de la empresa. En una opción quinta se encuentra las diferentes opciones de pago, en aras de que el cliente pueda pagar según y acorde a su necesidad. La sexta es garantizar seguimiento de la compra, es cuando la empresa permite al cliente estar informado de su compra. También está el formulario de venta, aunque en ocasiones no sea tan agradable llenar datos a la hora de comprar. Como octava se encuentra la competencia y las ofertas, en el mundo del internet existen diversas páginas y distintos vendedores del mismo producto. Los comentarios y valoraciones de otros usuarios es una más y trata de las referencias de personas reales para generar seguridad a las compras de clientes nuevos. Y por último está el servicio al cliente, este debe sentirse importante; se debe responder a tiempo y de la mejor manera posible. Y además se debe brindar una buena información a la hora de ofrecer cualquier producto (Antevenio, 
2016). Es importante que el vendedor siga esta clase de recomendaciones y factores claves para poder generar impacto en las ventas del mundo online.

Finalmente, el artículo permite conocer los factores (Ventajas y desventajas) que las estudiantes de la Universidad Francisco de Paula Santander Ocaña del programa de Administración de Empresas tienen en cuenta a la hora de comprar en el mundo virtual.

\section{Marco Teórico}

Se presentan a continuación algunas teorías claves para el estudio:

2.1 Teoría clásica. Al hablar de compras por internet, también se debe tocar el tema de ventas por ella misma; ya que no existirían los vendedores, sin los compradores. Las empresas en el pasado no podían acceder a las compras por internet por el motivo de que aún la tecnología no se los permitía, entonces ejecutaban otra clase de estrategias, pero hoy en día se tiene la posibilidad de manejar la dinámica de la compra por medio online, que permite comunicación entre partes lejanas, relaciones entre vendedores y compradores que por motivos de distancia no pueden ejercer el comercio físicamente. Por esta razón es necesario, dar a conocer los pasos que debe seguir una empresa para que sea exitosa; esto son la planificación, diseñar un plan de acción para el mañana, organización, brindar y movilizar recursos para la puesta en marcha del plan, dirección, dirigir, seleccionar y evaluar a los empleados con el propósito de lograr el mejor trabajo para alcanzar lo planificado, coordinación: integración de los esfuerzos y aseguramiento de que se comparta la información y se resuelvan los problemas y control: garantizar que las cosas ocurran de acuerdo con lo planificado y ejecución de las acciones correctivas necesarias de las desviaciones encontradas (Fayol, 1916). Puesto que de la misma manera en que se diseña una empresa físicamente, se hace virtualmente, ya que es importante llevar los principios para que de ese modo todo quede calculado y lo único que falte es ponerle la chispa de innovación con la plataforma online y quede por fin el proyecto completo.

2.2 Teoría del Long Tail. Se refiere a la manera en que actualmente se manejan algunos de los buscadores para generar compras. Ya que existen herramientas en las cuales el cliente no está enterado y por consiguiente no es su deber estarlo. Las compras por internet se han convertido en un método de facilidad con respecto al cliente y al vendedor, eso que de manera efectiva es posible realizar compras desde provincias, ciudades y países alejados del mismo. Existen diferentes maneras de adquirir el producto, una de ellas es por la opción de páginas web, pero requiere de algunos requisitos desde la mirada del vendedor para que sea posible sacar el mayor provecho a tal medio de venta (Ecommaster, 2015).

\section{Metodología}

La investigación fue de tipo descriptiva con enfoque cuantitativo, puesto que al finalizar y obtener resultados se proporcionaron análisis de información estadística que permitieron arrojar datos para resolver hipótesis sobre los factores que influyen durante una compra virtual desde la percepción de las estudiantes del programa de Administración de empresas de la Universidad Francisco de Paula Santander Ocaña. El estudio es probabilístico, puesto que se cumplen dos condiciones, una de ellas es que todos los elementos de la población tienen una probabilidad mayor a cero de ser 
seleccionados en la muestra y también la probabilidad de inclusión de cada elemento en la muestra se conoce de forma precisa (Ochoa, 2015) lo que significa que la investigación tiene más exactitud.

La población estuvo conformada por las estudiantes de género femenino del programa de administración de empresas de la universidad Francisco de Paula Santander, la cual está conformada por 371 estudiantes. Por otra parte, se aplicó el instrumento a 58 estudiantes, luego de ejecutada la muestra finita.

Por último, se puede resaltar que como técnica se utilizó una encuesta con el fin de obtener información medible, exacta y directa que no pudiera ser manipulada.

\section{Resultados}

El objetivo general de la investigación tiene como propósito identificar la dinámica del comercio virtual desde la participación de la juventud femenina de Ocaña, Norte de Santander. Con lo dicho, se presentan los siguientes resultados.

Tabla 1. Compras online por las estudiantes

\begin{tabular}{lcc}
\hline & Frecuencia & Porcentaje \\
\hline $\mathrm{Si}$ & 37 & $64 \%$ \\
$\mathrm{No}$ & 21 & $36 \%$ \\
Total & 58 & $100 \%$
\end{tabular}

Nota. Estudiantes que realizan procesos de compra virtual. Fuente. Elaboración propia.

De acuerdo a la estadística obtenida, se puede observar que las personas si están haciendo uso de las ventas virtuales, lo que es buena señal para las empresas que están en este ejercicio o quieren ingresar. Se tiene que un $64 \%$ de la población ha comprado por internet, lo que señala que ya han experimentado esta dinámica.

Por otra parte, se reconocen los siguientes factores de mayor importancia de los estudiantes en los procesos de compra.

Tabla 2. Búsqueda sencilla y manejo de las redes sociales

\begin{tabular}{lll}
\hline & Frecuencia & Porcentaje \\
\hline Siempre & 38 & $66 \%$ \\
A veces & 20 & $34 \%$ \\
Nunca & 0 & $0 \%$ \\
Total & 58 & $100 \%$ \\
\hline
\end{tabular}

Nota. Se presenta el factor de búsqueda e interacción fácil en la plataforma. Fuente. Elaboración propia.

Partiendo de los datos asegurados por el instrumento, se puede observar que uno de los factores que siempre incide es la búsqueda y manejo sencillo de la página para conocer el producto o servicio. Es decir, se puede ver que el porcentaje más notable es siempre con un $66 \%$. Seguidamente, el $34 \%$ con algunas veces.

Tabla 3. Información suficiente sobre el producto

\begin{tabular}{lll}
\hline & Frecuencia & Porcentaje \\
\hline Siempre & 46 & $79 \%$ \\
A veces & 12 & $21 \%$ \\
Nunca & 0 & $0 \%$ \\
Total & 58 & $100 \%$ \\
\hline
\end{tabular}

Nota. Se presenta el factor de información completa y concisa para adquirir un producto o servicio de las plataformas. Fuente. Elaboración propia.

Con relación a los resultados arrojados, la información específica del producto $y$ servicio es el factor más influyente que ven al momento de adquirir una compra por internet 
con un $79 \%$, por otro lado, el $21 \%$ aceptó que generalmente toman como referencia este factor para decidir sobre comprar tal producto. Dentro del proceso investigativo se conocen las desventajas al comprar por internet según la percepción de las estudiantes:

Tabla 4. Falta de comunicación y relación personal

\begin{tabular}{lll}
\hline & Frecuencia & Porcentaje \\
\hline $\mathrm{Si}$ & 36 & $62 \%$ \\
No & 22 & $38 \%$ \\
Total & 58 & $100 \%$ \\
\hline
\end{tabular}

Nota: En la tabla se puede observar una de las desventajas que generan arrepentimiento en la compra o a la hora de comprar por medio online. Fuente. Elaboración propia.

Antes se mencionaban las ventajas, se debe indicar que, también se presentan aspectos desfavorables, y una de ella es la falta de comunicación que puede haber en la compra, ya que no es posible establecer lazos cara a cara y existe la posibilidad además que sea un robo, por tanto, un $62 \%$ la población apunta que es una desventaja de las compras por internet.

Tabla 5. Imposibilidad de interactuar con el producto antes de la compra

\begin{tabular}{lll}
\hline & Frecuencia & Porcentaje \\
\hline $\mathrm{Si}$ & 53 & $91 \%$ \\
$\mathrm{No}$ & 5 & $9 \%$ \\
Total & 58 & $100 \%$ \\
\hline
\end{tabular}

Nota: En la tabla se puede observar una de las desventajas que generan arrepentimiento en la compra o a la hora de comprar por medio online. Fuente: Fuente. Elaboración propia.

En la compra virtual las expectativas son altas, por ende, es un riesgo que se toma a la hora de decidir comprar por internet, es decir, solo se hace una ilusión por la imagen de publicidad que se observa desde la internet, con lo anterior, el $91 \%$ de las jóvenes señalan que la imposibilidad de probar antes de comprar es desventaja del medio online.

Tabla 5. Dispositivo para conectarse a internet

\begin{tabular}{lll}
$\begin{array}{l}\text { Es imprescindible tener } \\
\text { dispositivo para } \\
\text { conectarse a internet }\end{array}$ & Frecuencia & Porcentaje \\
\hline $\mathrm{Si}$ & 33 & $57 \%$ \\
$\mathrm{No}$ & 25 & $43 \%$ \\
Total & 58 & $100 \%$ \\
\hline
\end{tabular}

Nota: En la tabla se puede observar una de las desventajas que generan arrepentimiento en la compra o a la hora de comprar por medio online. Fuente. Elaboración propia.

E1 57\% apunta que es una desventaja, pero el $43 \%$ considera lo contrario. Pero dado el caso que la mayoría tiene la razón. La cifra se presenta debido a que la provincia de Ocaña la población presenta dificultades de conectividad.

Tabla 6. Inseguridad y miedo a los pagos fraudulentos a las estafas y el robo de la información personal (hackers)

\begin{tabular}{lll}
\hline & Frecuencia & Porcentaje \\
\hline $\mathrm{Si}$ & 51 & $88 \%$ \\
$\mathrm{No}$ & 7 & $12 \%$ \\
Total & 58 & $100 \%$ \\
\hline
\end{tabular}

Nota: En la tabla se puede observar una de las desventajas que generan arrepentimiento en la compra o a la hora de comprar por medio online. Fuente. Elaboración propia.

Uno de los principales casos por los que nace la desconfianza de comprar por internet, se denomina "miedo" al pensar que la compra es un fraude y lo único que resulte al final es un robo. El 88\% está de acuerdo con la 
afirmación expresa y el $12 \%$ considera que existen empresas responsables y con políticas de rectitud, seguridad y respeto al cliente.

\section{Conclusiones}

Hoy por hoy la relación del comercio se establece entre un comprador más informado y un vendedor que debe adaptarse a estos paradigmas comunicacionales para que se pueda crear o avanzar en el proceso de empoderamiento comercio virtual, pensando siempre en satisfacer las expectativas del cliente, y así generar una ventaja competitiva para que el vendedor logre cumplir los objetivos empresariales.

Se pudieron observar claramente los factores que el cliente tiene en cuenta a la hora de ejecutar la compra. Primero que todo se observó que ellos identifican algunos de los principios evaluativos que influyen en la compra, los cuales son importantes para que sean adaptados por el vendedor. (Gómez, 2011) una de las cosas que más afecta las organizaciones y especialmente las Pymes, es la extrema personalización a las necesidades de cada tipo de cliente. La comunidad desde la plataforma está interesa en conocer todo sobre el producto y la facilidad de ingresar y familiarizarse con la misma sea sencilla.

Según Taváres (2013) la evaluación de experiencias ajenas es un buen punto de referencia para elegir qué queremos hacer y qué no; pero nunca debe ser un elemento paralizante para tomar acción. Al estudiar las ventajas y desventajas de las compras por internet, se comprende que los canales de comunicación deben centrarse a ofrecer información de seguridad y condiciones para la compra y adquisición del producto.
Finalmente, la venta virtual se considera una ventaja de competitividad integral desde los años 2000, frente a sus competidores, es decir, se encamina un sistema organizacional moderno que trata de satisfacer completamente a los consumidores, por ende, el tema de cumplir las expectativas del cliente sigue permaneciendo desde la virtualidad, ya que se está generando una relación social sobre el mercado (Moliner y Berenguer, 2011). Con lo anterior, el estudio permite considerar que las desventajas del ecommerce obtenidas por el estudio deben ser factores claves para generar estrategias $y$ promover un acercamiento de productos potenciales donde el cliente tenga la posibilidad de conocerlos y sentir conformidad al momento de adquirir.

\section{Referencias}

Antevenio. (2016). Antevenio- anticipation emarketing. Obtenido de 10 factores que influyen en la decisión de compra online:

https://www.antevenio.com/blog/201 6/10/10-factores-que-influyen-en-ladecision-de-compra-online/

Kutchera, J., García, H., \& Fernandez, A. (2014). EXITO-SU ESTRATEGIA DE MARKETING DIGITAL EN 5

PASOS. México: Grupo editorial patria.

Ecommaster. (09 de 04 de 2015). Ecommaster. Obtenido de Ecommaster: https://ecommaster.es/estrategia-debranding-para-ecommerce

Henry, F. ((1841-1925)). Teoría clásica de la administración. Obtenido de 
https://www.aulafacil.com/cursos/ad ministracion/de-empresas/teoriaclasica-de-la-administracion-fayol119660

Ochoa, C. (27 de febrero de 2015). netquest. Obtenido de Muestreo probabilístico o no probabilístico: https://www.netquest.com/blog/es/bl og/es/muestreo-probabilistico-o-noprobabilistico-ii

Gómez, D. (23 de octubre de 2011). BIENPENSADO. Obtenido de Cómo adaptarse a cada cliente y ser rentable: https://bienpensado.com/comoadaptarse-al-cliente-y-ser-rentable/

Tavárez Soriano Juan Dionicio. (2013, Mayo 16). Importancia de aprender de la propia experiencia. Recuperado de https://www.gestiopolis.com/importa ncia-de-aprender-de-la-propiaexperiencia/

Moliner, B., \& Berenguer, G. (2011). El efecto de la satisfacción del cliente en la lealtad: aplicación en establecimientos minoristas. Cuadernos de administración, 103. 